\title{
NEIGHBOR SUM DISTINGUISHING TOTAL CHROMATIC NUMBER OF PLANAR GRAPHS WITHOUT 5-CYCLES ${ }^{1}$
}

\author{
Xue Zhao \\ AND \\ Chang-QIng Xu ${ }^{2}$ \\ School of Science, \\ Hebei University of Technology \\ Tianjin 300401, P.R. China \\ e-mail: zhaoxhxy@163.com \\ chqxu@hebut.edu.cn
}

\begin{abstract}
For a given graph $G=(V(G), E(G))$, a proper total coloring $\phi: V(G) \cup$ $E(G) \rightarrow\{1,2, \ldots, k\}$ is neighbor sum distinguishing if $f(u) \neq f(v)$ for each edge $u v \in E(G)$, where $f(v)=\sum_{u v \in E(G)} \phi(u v)+\phi(v), v \in V(G)$. The smallest integer $k$ in such a coloring of $G$ is the neighbor sum distinguishing total chromatic number, denoted by $\chi_{\Sigma}^{\prime \prime}(G)$. Pilśniak and Woźniak first introduced this coloring and conjectured that $\chi_{\Sigma}^{\prime \prime}(G) \leq \Delta(G)+3$ for any graph with maximum degree $\Delta(G)$. In this paper, by using the discharging method, we prove that for any planar graph $G$ without 5-cycles, $\chi_{\Sigma}^{\prime \prime}(G) \leq$ $\max \{\Delta(G)+2,10\}$. The bound $\Delta(G)+2$ is sharp. Furthermore, we get the exact value of $\chi_{\Sigma}^{\prime \prime}(G)$ if $\Delta(G) \geq 9$.
\end{abstract}

Keywords: neighbor sum distinguishing total coloring, discharging method, planar graph.

2010 Mathematics Subject Classification: 05C15.

\section{REFERENCES}

[1] J.A. Bondy and U.S.R. Murty, Graph Theory with Applications (North-Holland, New York-Amsterdam-Oxford, 1982).

\footnotetext{
${ }^{1}$ This work was supported by NSFC (No.11671232), HNSF(No.A2015202301) and HU STP (No.ZD2015106, QN2017044).

${ }^{2}$ Corresponding author.
} 
[2] X. Cheng, D. Huang, G. Wang and J. Wu, Neighbor sum distinguishing total colorings of planar graphs with maximum degree $\Delta$, Discrete Appl. Math. 190-191 (2015) 34-41. doi:10.1016/j.dam.2015.03.013

[3] A. Dong and G. Wang, Neighbor sum distinguising total colorings of graphs with bounded maximum average degree, Acta Math. Sin. (Engl. Ser.) 30 (2014) 703-709. doi:10.1007/s10114-014-2454-7

[4] L. Ding, G. Wang and G. Yan, Neighbor sum distinguising total colorings via the Combinatorial Nullstellensatz, Sci. China Math. 57 (2014) 1875-1882. doi:10.1007/s11425-014-4796-0

[5] S. Ge, J. Li and C. Xu, Neighbor sum distinguishing total chromatic number of planar graphs without 4-cycles, Util. Math. 105 (2017) 259-265.

[6] S. Ge, J. Li and C. Xu, Neighbor sum distinguishing total coloring of planar graphs without 5-cycles, Theoret. Comput. Sci. 689 (2017) 169-175. doi:10.1016/j.tcs.2017.05.037

[7] H. Li, L. Ding, B. Liu and G. Wang, Neighbor sum distinguishing total colorings of planar graphs, J. Comb. Optim. 30 (2015) 675-688. doi:10.1007/s10878-013-9660-6

[8] J. Li, S. Ge and C. Xu, Neighbor sum distinguishing total colorings of planar graphs with girth at least 5, Util. Math. 104 (2017) 115-121.

[9] H. Li, B. Liu and G. Wang, Neighbor sum distinguishing total colorings of $K_{4}$-minor free graphs, Front. Math. China 8 (2013) 1351-1366. doi:10.1007/s11464-013-0322-x

[10] Q. Ma, J. Wang and H. Zhao, Neighbor sum distinguishing total colorings of planar graphs without short cycles, Util. Math. 98 (2015) 349-359.

[11] M. Pilśniak and M. Woźniak, On the total-neighbor-distinguishing index by sums, Graphs Combin. 31 (2015) 771-782. doi:10.1007/s00373-013-1399-4

[12] C. Qu, G. Wang, J. Wu and X. Yu, On the neighbor sum distinguishing total coloring of planar graphs, Theoret. Comput. Sci. 609 (2016) 162-170. doi:10.1016/j.tcs.2015.09.017

[13] C. Qu, G. Wang, G. Yan and X. Yu, Neighbor sum distinguishing total choosability of planar graphs, J. Comb. Optim. 32 (2016) 906-916. doi:10.1007/s10878-015-9911-9

[14] H. Song, W. Pan, X. Gong and C. Xu, A note on the neighbor sum distinguishing total coloring of planar graphs, Theoret. Comput. Sci. 640 (2016) 125-129. doi:10.1016/j.tcs.2016.06.007

[15] H. Song and C. Xu, Neighbor sum distinguishing total chromatic number of $K_{4}$ minor free graph, Front. Math. China 12 (2017) 937-947. doi:10.1007/s11464-017-0649-9 
[16] H. Song and C. Xu, Neighbor sum distinguishing total coloring of planar graphs without 4-cycles, J. Comb. Optim. 34 (2017) 1147-1158.

doi:10.1007/s10878-017-0137-x

[17] J. Wang, J. Cai and Q. Ma, Neighbor sum distinguishing total choosability of planar graphs without 4-cycles, Discrete Appl. Math. 206 (2016) 215-219.

doi:10.1016/j.dam.2016.02.003

[18] J. Wang, J. Cai and B. Qiu, Neighbor sum distinguishing total choosability of planar graphs without adjacent triangles, Theoret. Comput. Sci. 661 (2017) 1-7. doi:10.1016/j.tcs.2016.11.003

[19] J. Wang, Q. Ma and X. Han, Neighbor sum distinguishing total colorings of triangle free planar graphs, Acta Math. Sin. (Engl. Ser.) 31 (2015) 216-224. doi:10.1007/s10114-015-4114-y

[20] D. Yang, X. Yu, L. Sun, J. Wu and S. Zhou, Neighbor sum distinguishing total chromatic number of planar graphs with maximum degree 10, Appl. Math. Comput. 314 (2017) 456-468.

doi:10.1016/j.amc.2017.06.002

[21] J. Yao, X. Yu, G. Wang and C. Xu, Neighbor sum distinguishing total coloring of 2-degenerate graphs, J. Comb. Optim. 34 (2017) 64-70. doi:10.1007/s10878-016-0053-5

[22] J. Yao, X. Yu, G. Wang and C. Xu, Neighbor sum (set) distinguishing total choosability of d-degenerate graphs, Graphs Combin. 32 (2016) 1611-1620. doi:10.1007/s00373-015-1646-y

Received 5 December 2017 Revised 8 February 2018 Accepted 7 March 2018 